\title{
On the effect of cosmic rays in bolometric cosmic microwave background measurements from the stratosphere
}

\author{
S. Masi ${ }^{1,2}$, E. Battistelli ${ }^{1,2}$, P. de Bernardis ${ }^{1,2}$, L. Lamagna ${ }^{1,2}$, F. Nati ${ }^{1,2}$, L. Nati $^{1,2}$, P. Natoli ${ }^{3}$, \\ G. Polenta ${ }^{4,5}$, and A. Schillaci ${ }^{1,2}$ \\ 1 Dipartimento di Fisica, Università di Roma "La Sapienza", Roma, Italy \\ e-mail: silvia.masi@roma1.infn.it \\ 2 INFN Sezione di Roma 1, Roma, Italy \\ 3 Dipartimento di Fisica, Università di "Tor Vergata”, Roma, Italy \\ 4 Agenzia Spaziale Italiana - ASI Science Data Center, Frascati, Italy \\ 5 INAF - Osservatorio Astronomico di Roma, Monte Porzio Catone, Italy
}

Received 14 January 2010 / Accepted 11 May 2010

ABSTRACT

\begin{abstract}
Context. Precision measurements of the anisotropy of the cosmic microwave background (CMB) are able to detect low-level nonGaussian features caused by either topological defects or the inflation process. These measurements are becoming feasable with the development of large arrays of ultra-sensitive bolometric detectors and their use in balloon-borne or satellite missions. However, the space environment includes a population of cosmic rays (CRs), which produce spurious spikes in bolometric signals.

Aims. We analyze the effect of CRs on the measurement of CMB anisotropy maps and the estimate of cosmological non-Gaussianity and angular power spectra of the CMB.

Methods. Using accurate simulations of noise and CR events in bolometric detectors, and de-spiking techniques, we produce simulated measured maps and analyze the Gaussianity and power spectrum of the maps for different levels and rates of CR events.

Results. We find that a de-spiking technique based on outlier removal in the detector signals contributing to the same sky pixel is effective in removing CR events larger than the noise. However, low level events hidden in the noise produce a positive shift of the average power signal measured by a bolometer, and increase its variance. If the number of hits per pixel is large enough, the data distribution for each sky pixel is approximately Gaussian, but the skewness and the kurtosis of the temperatures of the pixels indicate the presence of some low-level non-Gaussianity. The standard noise estimation pipeline produces a positive bias in the power spectrum at high multipoles.

Conclusions. In the case of a typical balloon-borne survey, the CR-induced non-Gaussianity will be marginally detectable in the membrane bolometer channels, but be negligible in the spider-web bolometer channels. In experiments with detector sensitivity better than $100 \mu \mathrm{K} / \sqrt{\mathrm{Hz}}$, in an environment less favorable than the earth stratosphere, the CR-induced non-Gaussianity is likely to significantly affect the results.
\end{abstract}

Key words. cosmic background radiation - instrumentation: detectors - space vehicles - methods: data analysis

\section{Introduction}

Information about the early Universe is encoded in the primary anisotropy of the CMB. While the Gaussian fluctuations expected in the adiabatic inflationary scenario beautifully fit the available power spectrum data (see e.g. Nolta et al. 2009; and Komatsu et al. 2010), non-Gaussian signals are also expected at a lower level in the maps, due to non-linearities in the inflation potential (see e.g. Verde et al. 2000) and/or the presence of topological defects (see e.g. Kaiser \& Stebbins 1984). In addition, non-Gaussian secondary anisotropies are imprinted in the postrecombination universe because of the interaction of CMB photons with the large-scale structures present in the Universe, and the emission of Galactic and extragalactic sources. The study of primordial non-Gaussianity can in principle allow to confirm and select an inflation model, but the signal to be detected is very small. For this reason, it is essential to ensure that the measurement system does not introduce non-Gaussian features into the data.

Arrays of microwave detectors feature high mapping speed and are now starting to operate at the focus of large telescopes (see e.g. Sayers et al. 2009; Siringo et al. 2009; Carlstrom et al. 2009; Wilson et al. 2008; Swetz et al. 2008) or in CMB polarimeters (see e.g. Hinderks et al. 2009; Yoon et al. 2006; Kuo et al. 2006; Samtleben et al. 2007). Transition edge superconducting (TES) bolometric detectors, involving only litographic fabrication techniques, are easier to replicate in large arrays, and extremely sensitive.

To fully exploit their sensitivity, the radiative background has to be minimized. In this sense, the optimal solution is to use bolometric detectors in space, possibly feeding them by means of a cryogenic telescope or optical system. The bolometers of the HFI instrument on the Planck mission (Holmes et al. 2008) and those of the PACS and SPIRE instruments on the Herschel mission (Billot et al. 2009; Schultz et al. 2008) represent a first step in this direction.

Being extremely sensitive to any form of energy deposited on their absorber and thermistors, bolometers are also sensitive to cosmic rays. The energy deposited by a single $\mathrm{MeV}$ proton in a cryogenic bolometer is much higher than the typical level of the noise (see e.g. Caserta et al. 1990): if an amount of energy $\Delta E$ is deposited in a bolometer in a time much shorter than the 
bolometer time constant, the temperature rise of the bolometer will be $\Delta T=\Delta E / C$, where $C$ is the heat capacity of the detector. This peak level will be achieved sooner than one time constant; the subsequent decay, instead, will be regulated by the time constant. The typical noise of a bolometer corresponds to physical temperature fluctuations much lower than $\Delta E / C$, so most CRinduced spikes will be evident in the bolometer time-ordereddata.

To allow the operation of bolometers in unprotected environments (such as sub-orbital or orbital space instruments), special low cross-section bolometers have been developed (spider-webs: Mauskopf et al. 1997; wire-grids: Jones et al. 2003). An additional benefit of these low-cross-section detectors is the reduced heat capacity with respect to solid-absorber or membraneabsorber ones, resulting in a shorter time constant.

In a polar stratospheric balloon flight, the rate of cosmicray events in low cross-section (spider-web) bolometers cooled to $0.3 \mathrm{~K}$ is on the order of $0.1 \mathrm{~Hz}$ (Masi et al. 2006), which is about 20 times less than the rate of events for solid/membraneabsorber bolometers at the same temperature in the same environment. The events are produced either by primary CR interacting with the detectors, or by secondary particles, including showers of electrons and bremmstrahlung-produced gammas, resulting from the interactions of primary cosmic rays with the metal surrounding the bolometric detector. Lower temperature detectors of basically the same geometry are affected by lower noise, such that the rate of $\mathrm{CR}$ events above the noise level is even higher; this effect is in part mitigated by their more rapid response. Rates between $0.02 \mathrm{~Hz}$ and $0.3 \mathrm{~Hz}$ have been measured for spider-web bolometers cooled to $0.1 \mathrm{~K}$ in similar stratospheric conditions (see Macias-Perez et al. 2007). The promising kinetic inductance detectors (KIDs), currently under development, are also sensitive to CRs, at least if the substrate is solid $\mathrm{Si}$ or sapphire. Anyway, their very fast response reduces significantly the fraction of contaminated data. Moreover, their sensitivity to CRs can be strongly reduced by depositing the KID resonator on a membrane. Coherent radiometers, using macroscopic thermally stable components, are much less prone to $\mathrm{CR}$ glitches.

Balloon-borne missions, using bolometer arrays, currently under development, include among others the EBEX CMB polarimeter (Oxley et al. 2004), the SPIDER experiment to detect CMB polarization at larger angular scales (Crill et al. 2008), the OLIMPO telescope, mainly devoted to high frequency observations of the Sunyaev-Zeldovich effect (Nati et al. 2007), and the BLAST/BLASTPOL telescope devoted to far infrared cosmological and Galactic studies (Pascale et al. 2008; Marsden et al. 2008). Given the high mapping speed of large arrays of bolometers and the efficient and relatively cheap access to space provided by stratospheric balloons, we expect these efforts to continue in the future. It is thus relevant to focus on the stratospheric case.

The data from a bolometric detector can be cleaned by detecting the spikes and flagging the corresponding section of data as unusable for additional analysis. For filtering purposes, the masked data can be filled with constrained realizations of noise with the same properties as the surrounding data (see e.g. Masi et al. 2006). However, in the absence of independent cosmic rays monitors, a number of low level events will remain unidentified, hidden in the noise. If the bolometer is non-ideal, and features an additional long time constant, the effect of CR can be an increase in $1 / \mathrm{f}$ noise. In this paper, we focus on the undetected cosmic rays hits and study how they can affect the analysis of data aimed at measuring CMB anisotropy and non-Gaussianity.

\section{Simulation of bolometer time-ordered data}

To study the effect of CR events, we simulated first time-streams of bolometer data in the absence of sky signals, i.e., we added CR events to a Gaussian realization of noise $G(t)$ with a given standard deviation $\sigma$ and null average. The time-ordered samples of bolometer noise are built as a Gaussian realization, filtered with a first-order low-pass filter simulating the thermal response of the detector.

We considered two reference cases with different bolometer time constants and CR rates: case $R_{1}$ has a rate of $2 \mathrm{~Hz}$ for a slow, membrane absorber bolometer at $0.3 \mathrm{~K}(\tau=70 \mathrm{~ms})$, and case $R_{2}$ is a low-cross-section bolometer (spider-web) with a rate of $0.1 \mathrm{~Hz}$ and a time constant of $\tau=10 \mathrm{~ms}$.

The waiting times for CR events follow an exponential distribution of rate $R$

$P(t)=R \mathrm{e}^{-R t}, t>0$.

The amplitudes $A$ of the pulses induced by CRs are assumed to follow an exponential distribution with average $\langle A\rangle$. The exact shape of this distribution for a given experiment depends on the spectra of primary cosmic rays in the environment of operation, on the distribution and characteristics of the material surrounding the bolometer, on the shape of the sensitive element of the bolometer itself, and on the angular distribution of the incoming cosmic rays in the restframe of the bolometer sensor. The important parameter, however, is the fraction of events that have amplitudes lower than say $3 \sigma$ : these are likely to remain hidden in the noise. In the case of the exponential distribution that we selected, this fraction is $1-\exp (-3 \sigma /\langle A\rangle)$, which is $\sim 26 \%$ if $\langle A\rangle / \sigma=10$.

The shape of each pulse is modeled as a sudden level jump of amplitude $A$, followed by an exponential decay with a time constant $\tau$ :

$D(t)=A \mathrm{e}^{-t / \tau}$

For 10 time constants after the CR event, the simulated data are obtained as $S(t)=D(t)+G(t)$, whereas elsewhere the simulated data are simply $G(t)$. In our initial tests, we do not include sky signals in the simulation.

We simulated chunks of $10 \mathrm{~h}$ of data, to obtain a sufficient number of events: we have an average of 72000 (3600) events per chunk in case $R_{1}\left(R_{2}\right)$. Our full set of simulations includes 10000 simulated chunks.

In Figs. 1 and 2, we show sample subsections of simulated data. Large CR spikes are evident, but smaller ones remain hidden in the noise.

\section{Despiking the time-ordered data}

Evident CR events (with amplitudes larger than say $4 \sigma$ ) can be easily identified and removed. To remove smaller CR spikes, one can take advantage of the peculiar shape of these signals. The exact shape depends on the bolometer configuration (single or double time constant, or even more complex response) and on the details of the front-end electronics, amplifying the $\mathrm{nV}$-level bolometer signals. In our simulations, we used a simple exponential decay, but it would not be difficult to extend our analysis to more complex shapes, once the impulsive response of the system is known from calibrations. 


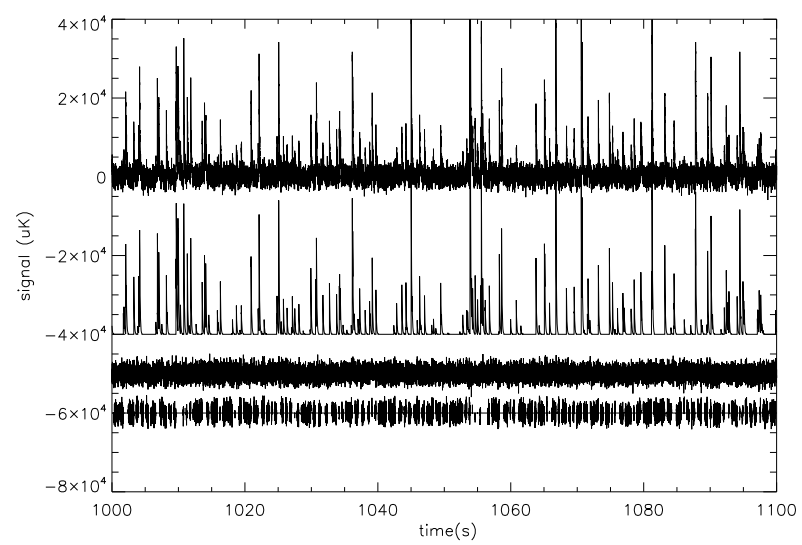

Fig. 1. Top line: sample subsection of simulated bolometer data $S(t)$ (converted in $\mu K_{\mathrm{CMB}}$ ) resulting from the sum of spikes from $\mathrm{CR}$ events $D(t)$ (middle top line, offset $-40 \mathrm{mK}$ for clarity of visualization) and bolometer noise $G(t)$ (middle bottom line, offset $-50 \mathrm{mK}$ ). In this simulation, the signal is sampled at $200 \mathrm{~Hz}, \sigma=1.4 \mathrm{mK}, \tau=0.07 \mathrm{~s}$, $R=2.0 \mathrm{~Hz}$, and $\langle A\rangle=10 \mathrm{mK}$ (case $R_{1}$ ). Comparing the top and middle lines, it is evident that many CR events remain hidden in the noise and are very difficult to identify. In the bottom line (offset $-60 \mathrm{mK}$ ), we plot the data de-spiked following the pixel-based procedure described in the text. The missing data have been flagged as unusable because they are within 5 time constants of a detected spike.

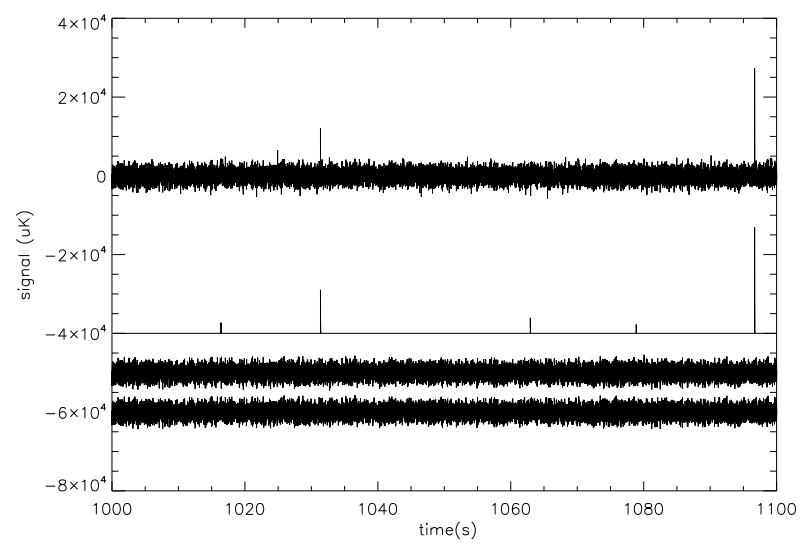

Fig. 2. Same as Fig. 1, with $\tau=0.01 \mathrm{~s}, R=0.1 \mathrm{~Hz}$, and $\langle A\rangle=10 \mathrm{mK}$ (case $R_{2}$ ).

In the simulations described in this section, we did not include any sky signal. With a typical $200 \mathrm{~Hz}$ sampling rate and $100 \mu \mathrm{K} / \sqrt{\mathrm{Hz}} \mathrm{NET}$, the standard deviation in the bolometer noise is on the order of $1400 \mu \mathrm{K}$, while the standard deviation in the CMB anisotropy is on the order of $100 \mu \mathrm{K}$. It is thus very unlikely that CMB signals can affect the performance of any despiking procedure. Diffuse Galactic emission can reach levels on the order of or higher than $1000 \mu \mathrm{K}$ only in the Galactic plane, which is not useful for CMB studies. Point sources are also masked in sensitive CMB searches.

A standard method for identifying events embedded in noise is to search for correlations between the noisy time ordered data and an event template. To estimate the correlation, we computed the normalized convolution

$C(t)=\frac{\int_{0}^{10 \tau} S(t+u) D(u) \mathrm{d} u}{\int_{0}^{10 \tau} D(u) \mathrm{d} u}$.

In the absence of noise, this correlation is positive for all times $t$ where a CR event contaminates the signal. Noise induces
Table 1. Fraction of true detections $\left(f_{\mathrm{t}}\right)$ and false detections $\left(f_{\mathrm{f}}\right)$ versus threshold level $T$ for the convolution-based despiking method, for the two reference cases $\left(R_{1}\right.$ and $\left.R_{2}\right)$ (see text). Here $\langle A\rangle=10 \sigma$.

\begin{tabular}{ccccc}
\hline \hline Threshold $T$ & $f_{\mathrm{t}}\left(R_{1}\right)(\%)$ & $f_{\mathrm{f}}\left(R_{1}\right)(\%)$ & $f_{\mathrm{t}}\left(R_{2}\right)(\%)$ & $f_{\mathrm{f}}\left(R_{2}\right)(\%)$ \\
\hline $0.5 \sigma$ & 37 & 2.9 & 33 & 19 \\
$1 \sigma$ & 27 & 0.3 & 24 & 3.8 \\
$2 \sigma$ & 16 & 0.005 & 13 & 0.02 \\
\hline
\end{tabular}

fluctuations in $C(t)$ that, however, are smaller than the fluctuations in $S(t)$, so there is some advantage in using this estimator. We defined a positive threshold $T$ and assumed that all the samples with $C(t)>T$ are contaminated by CR events. To analyze the efficiency and accuracy of this CR detection method, we considered the contaminated samples as a function of the threshold level $T$, and computed the fractions $f_{t}$ and $f_{f}$. The parameter $f_{t}$ is the fraction of events that have $C(t)>T$ and $D(t)>D_{\min }$, where $D_{\min }=1 \mu \mathrm{K}$ : these are true detections of CR events. In contrast $f_{f}$ is the fraction of samples where $C(t)>T$ while $D(t)<D_{\min }$ : these are false detections caused by noise mimicking CR events. For an average amplitude of the CR events equal to 7 times the rms of the noise $(\langle A\rangle=10 \sigma)$, we found that $50 \%$ $(0.4 \%)$ of the samples are contaminated in case $R_{1}\left(R_{2}\right)$.

Owing to the noise, we found that the convolution-based method fails to identify a number of contaminated samples, and introduces a large number of false detections (see Table 1 for $\langle A\rangle=10 \sigma)$. To avoid a large number of false detections, we found that one has to raise the threshold level, although many $\mathrm{CR}$ events are then missed. The situation is even worse if the ratio $\langle A\rangle / \sigma$ is lowered.

\section{Pixel-space despiking}

In addition to the problems listed in the previous section, removing CR pulses directly from the time-ordered data is inadvisable, because of the risk of removing true sky signals (for example, fast signals produced by scans over point sources, such as planets used as calibrators).

A more effective strategy is to analyze the set of data samples contributing to the same sky pixel: for all of these samples, the sky signal is the same, so outliers must be caused by either detector noise or CR events. This is true in the absence of pointing errors. In areas with steep brightness gradients (for example near the Galactic plane), the combination of pixelization and pointing errors can lead to spurious glitches. We neglect these effects here, since they are not relevant to CMB studies.

We used a simple iterative procedure to remove outliers. For a given pixel $p$, the average $\langle S\rangle_{p}$ and the standard deviation $\sigma_{p}$, in all the contributing time-ordered signals $S_{k}$ were computed. For sample $k$, if the difference $S_{k}-\langle S\rangle_{p}$ was larger than $3 \sigma_{p}$, the sample was classified as an outlier, and removed. Detection of outliers was therefore performed in pixel space, but outliers were removed in the time domain. New values of both the average and variance were computed from the remaining samples of the set, and new outliers were identified and removed. This procedure was repeated until the average and the variance no longer changed (no or few new outliers are found).

In general, only a few iterations are needed to achieve convergence. We found that after 6 iterations the efficiency of this removal method is similar to that of the convolution-based one, yet with this method we do not remove true sky signals.

The despiked timelines are very similar to the noise-only time-lines, and their spectrum (in frequency domain) closely 


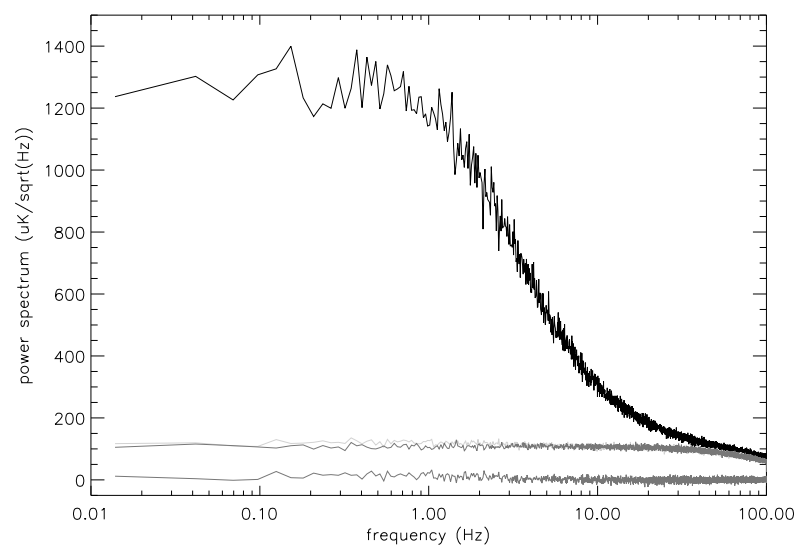

Fig. 3. Power spectrum of the data for case $R_{1}$ (top line), of the despiked data, and of the original noise-only data (middle lines). The despiking procedure does not introduce spectral features in the data, as is evident from the difference between the despiked spectrum and the original noise-only spectrum (bottom line).

resembles the noise spectrum, as shown in Fig. 3 for our worstcase $R_{1}$.

The average in each pixel converges to a positive value, while according to detector noise only it would be very close to zero. This is due to CR hits of amplitude smaller than the noise: these remain undetected and produce a positive bias. They also increase the standard deviation in the data contributing to the same pixel.

For this reason, the 1-point distribution of temperatures in pixels obtained after the averaging and the outlier-removal processes will be shifted towards positive values, and be broader than expected from instrument noise only.

After a CR event, the data are contaminated for a certain amount of time, depending on both the spike amplitude and the time constants of the system. Some of these data might be recovered by removing a best-fit spike profile from the timeline. However, due to noise, the fit will not be perfect. We prefer to be conservative, and flag as unusable all the data after a spike, for a period of 5 time constants. The fraction of flagged data is plotted in Fig. 4 for different CR rates and bolometer time constants. The obvious effect of CR events is thus a significant decrease in the redundancy of the maps. Only the use of fast detectors mitigates this problem. In the following, we focus on the second effect, i.e. the introduction of bias and non-Gaussian features in the maps.

We study two different cases. The first case is a targeted observation of a small map (about $1.5^{\circ} \times 1.5^{\circ}$ in size, with a $4^{\prime}$ FWHM resolution) for a relatively short time ( $10 \mathrm{~h}$ of integration for a single detector), such as the maps observed by the OLIMPO experiment to measure the Sunyaev-Zeldovich effect in well known clusters (Nati et al. 2007). The second is the observation of a large (about $10^{\circ} \times 10^{\circ}$ in size) deep survey of a clean region, with 7 days of integration. This is similar to the blind deep survey of CMB and undetected SZ clusters carried out by OLIMPO. We assume the same angular resolution for this survey as well.

In Fig. 5, we plot the 1-point distributions versus the iteration number in the case of observations of 4320 independent pixels (small map case), in our two reference cases, for a total integration time on the map of $10 \mathrm{~h}$. The results for the moments of these distributions are reported in Table 2.

It is clear that the outlier removal process is effective, since the 1-point distribution approaches a Gaussian distribution after

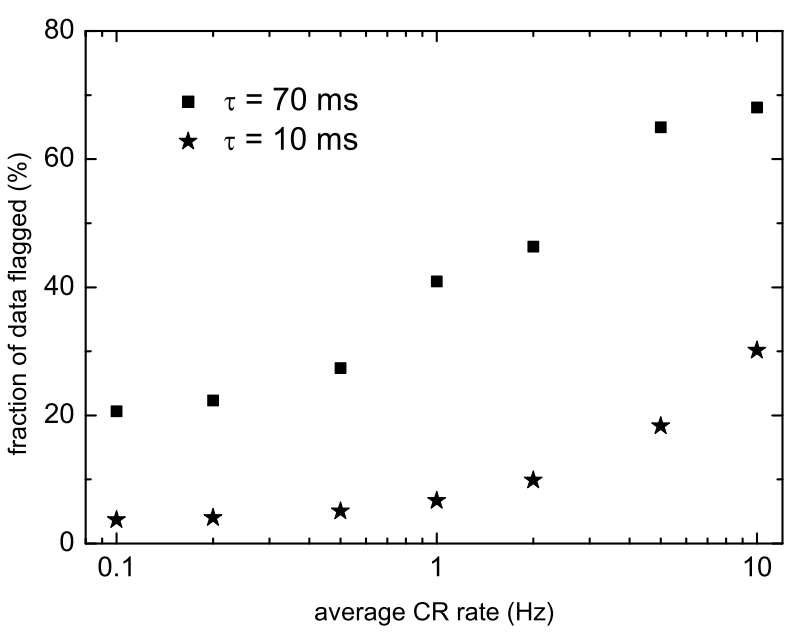

Fig. 4. Fraction of data flagged as contaminated by CR events, versus average $\mathrm{CR}$ rate, in a bolometric experiment. A detector noise of $100 \mu \mathrm{K} / \sqrt{\mathrm{Hz}}$ and an average amplitude of CR events of $10 \mathrm{mK}$ have been assumed. Squares refer to fast detectors (time constant of $10 \mathrm{~ms}$ ), stars refer to slow detectors (time constant of $70 \mathrm{~ms}$ ). A pixel-based de-spiking algorithm iteratively clipping all the data at more than $3 \sigma$ from the pixel average has been used. All data within 5 time constants of a CR event have been flagged.
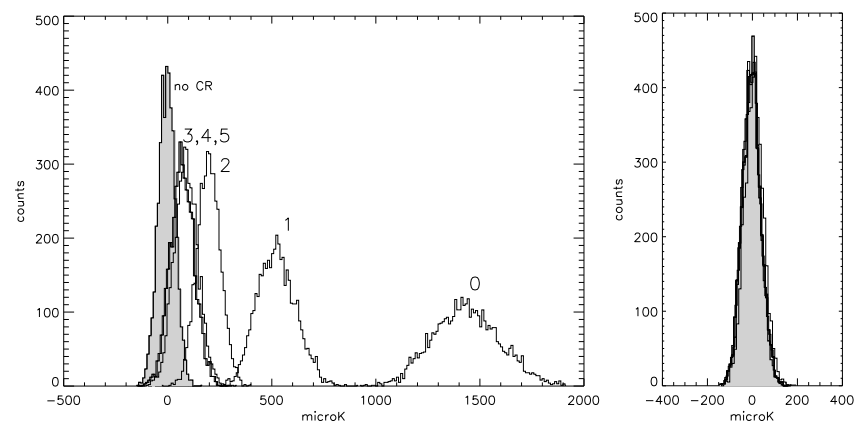

Fig. 5. Left: one-point distributions of pixel temperatures estimates $\langle S\rangle_{p}$, vs. despiking iteration. In this simulation, there is no sky signal, the detector NET is $100 \mu \mathrm{K} \sqrt{s}$, the time constant is $70 \mathrm{~ms}$, the sampling rate is $200 \mathrm{~Hz}(\sigma=1.4 \mathrm{mK})$, and the total integration time for 4320 pixels is $10 \mathrm{~h}$, in an environment producing an average amplitude of CR events of $10 \mathrm{mK}$ and an average rate of $2 \mathrm{~Hz}$ (case $R_{1}$ ). The distributions are labeled with the number of outlier removal iterations. After each iteration, the 1-point distribution moves to the left, approaching a Gaussian distribution but remaining shifted with respect to the distribution of detector noise without CR events (which is the leftmost histogram, hatched and labeled "no CR"). Right: here the time constant is $10 \mathrm{~ms}$, in an environment producing an average amplitude of CR events of $10 \mathrm{mK}$ and an average rate of $0.1 \mathrm{~Hz}$ (case $R_{2}$ ): the different distributions are hardly distinguishable.

a few iterations. The skewness and kurtosis of the 1-point distribution return to values consistent with zero, after the despiking process. This means that the number of low level spikes averaged in each pixel is large enough to produce a more Gaussian distribution, at least at the level of sensitivity considered here.

However, the positive bias and the increase in the variance in the estimated pixel temperatures caused by undetected CR persist, as is evident from Fig. 5 and Table 2.

If the average rate of CR hits is not constant during the observations of different pixels, this positive bias varies accordingly, introducing fake structures in the measured maps. A modulation of the CR rate can be introduced by the scanning strategy of 
Table 2. Parameters of the 1-point distribution of pixel temperature estimates, versus iteration of the pixel-based despiking, for cases $R_{1}$ and $R_{2}$ (observations of small maps, same conditions as in Fig. 5).

\begin{tabular}{ccccc}
\hline \hline Iteration & Average $(\mu \mathrm{K})$ & rms $(\mu \mathrm{K})$ & skewness & kurtosis \\
\hline no CR & $0.0 \pm 0.7$ & $39.6 \pm 0.4$ & $-0.001 \pm 0.038$ & $0.022 \pm 0.078$ \\
\hline $0\left(R_{1}\right)$ & $(1439 \pm 6)$ & $(159 \pm 4)$ & $(0.193 \pm 0.070)$ & $(0.06 \pm 0.15)$ \\
$1\left(R_{1}\right)$ & $(529 \pm 3)$ & $(92.3 \pm 2.1)$ & $(0.231 \pm 0.070)$ & $(0.11 \pm 0.15)$ \\
$2\left(R_{1}\right)$ & $(173.8 \pm 1.5)$ & $(59.1 \pm 0.8)$ & $(0.092 \pm 0.042)$ & $(0.059 \pm 0.087)$ \\
$3\left(R_{1}\right)$ & $(94.3 \pm 1.1)$ & $(56.0 \pm 0.6)$ & $(0.020 \pm 0.038)$ & $(0.029 \pm 0.076)$ \\
$4\left(R_{1}\right)$ & $(81.4 \pm 1.1)$ & $(57.4 \pm 0.6)$ & $(0.014 \pm 0.038)$ & $(0.029 \pm 0.076)$ \\
$5\left(R_{1}\right)$ & $(79.3 \pm 1.1)$ & $(57.9 \pm 0.6)$ & $(0.012 \pm 0.038)$ & $(0.029 \pm 0.077)$ \\
\hline $0\left(R_{2}\right)$ & $(12.5 \pm 0.7)$ & $(41.9 \pm 0.5)$ & $(0.069 \pm 0.039)$ & $(0.089 \pm 0.087)$ \\
$1\left(R_{2}\right)$ & $(1.1 \pm 0.7)$ & $(40.6 \pm 0.4)$ & $(0.001 \pm 0.037)$ & $(0.029 \pm 0.078)$ \\
$2\left(R_{2}\right)$ & $(0.8 \pm 0.7)$ & $(40.9 \pm 0.4)$ & $(0.001 \pm 0.037)$ & $(0.029 \pm 0.077)$ \\
$3\left(R_{2}\right)$ & $(0.8 \pm 0.7)$ & $(41.0 \pm 0.5)$ & $(0.001 \pm 0.037)$ & $(0.029 \pm 0.078)$ \\
$4\left(R_{2}\right)$ & $(0.8 \pm 0.7)$ & $(41.0 \pm 0.4)$ & $(0.001 \pm 0.037)$ & $(0.029 \pm 0.078)$ \\
$5\left(R_{2}\right)$ & $(0.8 \pm 0.7)$ & $(41.0 \pm 0.4)$ & $(0.001 \pm 0.037)$ & $(0.029 \pm 0.078)$ \\
\hline
\end{tabular}

Notes. Iteration 0 refers to the values without despiking. The first line reports the noise-only case (no CRs). The errors describe the dispersion in the results of 3000 simulations.

the instrument, which induces variations in the absorbing material in-between the CR flux and the bolometers, thus producing scan-synchronous systematic effects. For example, an asymmetric satellite spinning in the solar wind could introduce spurious dipole or higher order anisotropy (depending on the structure of the satellite) aligned to the solar wind direction. It would be very useful to include in the instrumental setup an independent CR flux monitor, as close as possible to the focal plane bolometers. The level of the spurious signal depends on the CR composition, flux and spectrum, on the cross-section of the bolometers, and on the structure of the instrument; this can be quantified only by focusing on the specific case.

Even if the CR flux is perfectly steady, the increase in the variance of the detected data for each pixel can bias the noise estimates, which are needed to estimate the power spectra. In the standard analysis procedure for CMB maps one estimates the noise from the data, assuming Gaussian noise, and then uses Gaussian Monte Carlo simulations to assess the bias in the power spectrum (see e.g. Hivon et al. 2002; Polenta et al. 2005).

In principle, this can bias the noise estimates, and, at a lower level, might affect the non-Gaussianity parameters relevant to cosmology.

These effects are studied in the following paragraphs, where we focus on observations of the larger survey, which is most useful to minimizing cosmic variance and measuring the power spectrum of the CMB and non-Gaussianity parameters. This $\sim 100$ square degrees map contains $N=860701.7^{\prime}$ pixels. A total integration time of 1 week is assumed.

We define a data timeline contaminated by spikes for the cases $R_{1}$ and $R_{2}$ described above (70 and $10 \mathrm{~ms}$ detector time constant, respectively). These are, respectively, a hard case and a mild case, from the point of view of CR contamination. We thus perform iterative despiking as described above, and analyze the resulting large maps.

\section{Tests of Gaussianity}

Primordial CMB anisotropies are known to have Gaussian distributions to leading order (see e.g. Komatsu et al. 2003; De Troia et al. 2003; Natoli et al. 2010). Small deviations from Gaussianity have however been predicted and, if observed, may be used to constrain inflationary models (see Bartolo et al. 2004, for a review).

Measurements of this non-Gaussian component are however hampered by several sources of instrumental or non instrumental (e.g., foreground contamination) systematic effects, which may induce spurious non-Gaussian signatures in the data.

In particular, undetected cosmic-ray hits add a non-Guassian contribution to detector noise, which is usually assumed to be otherwise normally distributed. Furthermore, the despiking procedures described above are based on clipping and may also alter the statistics of the dataset.

We first perform a Kolmogorov-Smirnov (KS) test on the pixel values for (1) five iteration despiking for the $R_{1}$ and $R_{2}$ and (2) no CR contamination, the latter to be used as the reference purely Gaussian dataset. The KS test (see Press et al. 1992) is a standard test to quantify the probability that a sample can be ascribed to a given distribution. It can also be used to reject the null hypothesis that two different samples are drawn from the same distribution. For each map, we compute the KS measure

$D_{\mathrm{obs}}=\max _{t}\left|S_{N}(t)-F(t)\right|$

where $t$ is the map temperature value, $F$ the cumulative of the Gaussian distribution function, and $S_{N}$ the empirical distribution function of a simulated noise map. We also define $Z_{\text {obs }}=D_{\text {obs }} \sqrt{N}$. The KS test provides a probability $P\left(Z>Z_{\text {obs }}\right)$ for a given map. In Fig. 6, we plot the KS probabilities obtained for the 140 Monte Carlo maps as histograms for the $R_{1}$ (top) and $R_{2}$ cases, along with their respective Gaussian (no CR but rescaled effective variance ${ }^{1}$ ) reference set (hatched). The $R_{1}$ case exhibits a clear deviation from its reference Gaussian counterpart, while in the case of $R_{2}$ this deviation is not at all evident. Even for $R_{1}$, any single map has (roughly) a $50 \%$ probability of being flagged as non-Gaussian by our KS analysis. The same considerations apply to the observed distribution of the $Z$ coefficients, shown in Fig. 7.

To quantify our detection of non-Gaussianity, we define two simple NG estimators: the normalized skewness $\left(S_{3}\right)$ and

\footnotetext{
1 The reference Gaussian dataset is different in the two cases because the noise realizations are different and their variances are corrected to account for the increase in noise caused by despiking; see the next section for further details.
} 

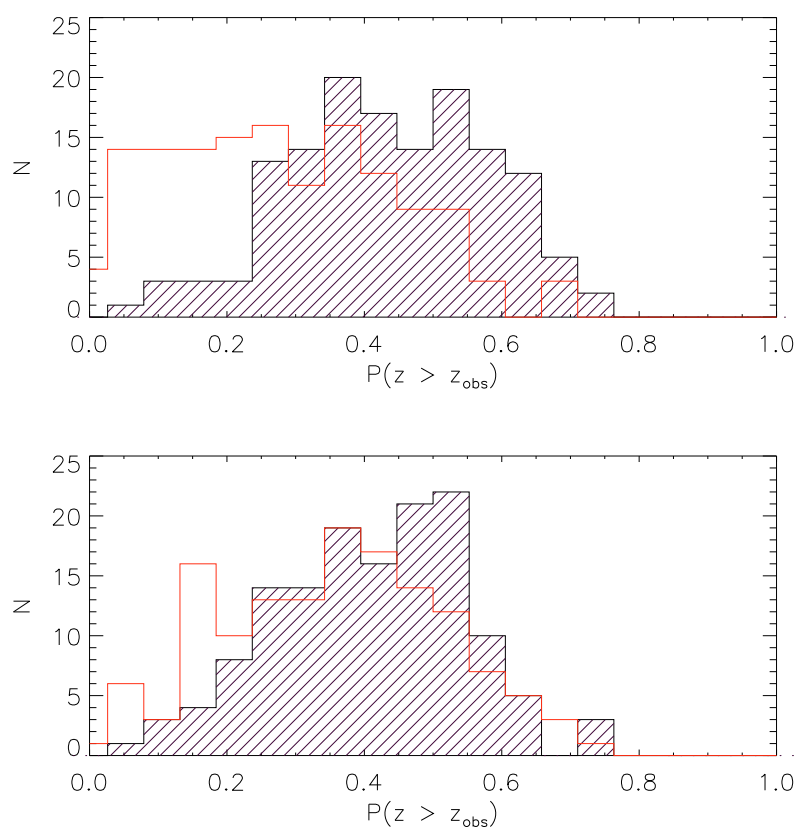

Fig. 6. Histogrammed probabilities of the KS test, for the $R_{1}$ (top) and $R_{2}$ (bottom) cases. The hatched histograms refer to the reference Gaussian sets.
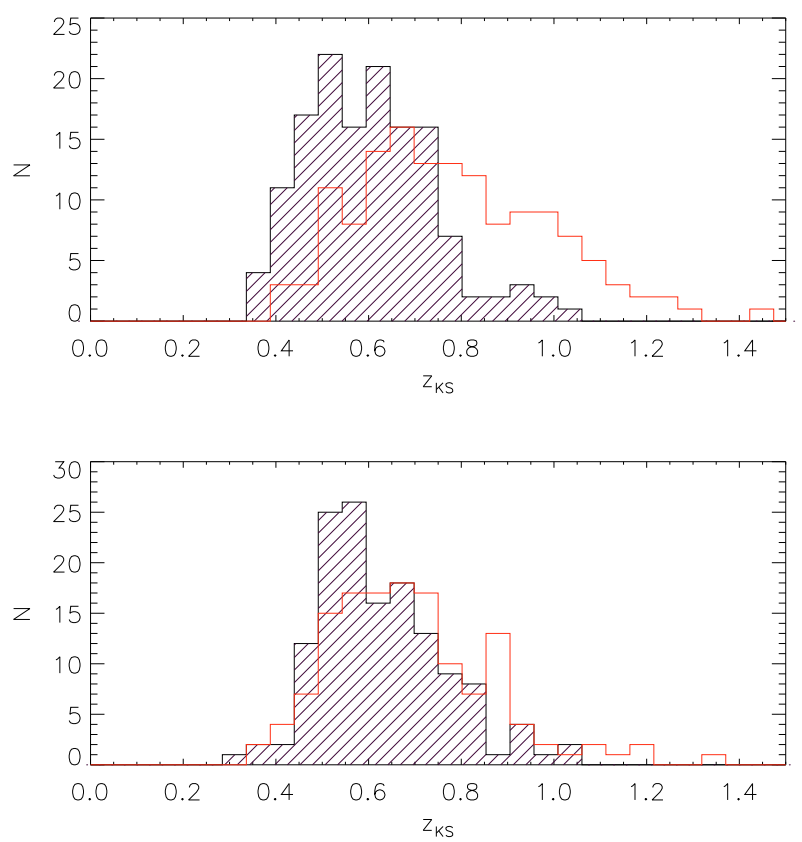

Fig. 7. Same as Fig. 6 but for the $Z_{\mathrm{obs}} \mathrm{KS}$ coefficients.

kurtosis $\left(S_{4}\right)$ of each map. The corresponding histograms are shown in Fig. 8, again for the cases $R_{1}, R_{2}$, and their respective reference Gaussian sets (hatched). We note how the $R_{2}$ case also exhibits deviation from Gaussianity in the case of the kurtosis. The empirical (histogrammed) distribution functions can themselves be subject to a KS test against their Gaussian reference sets: in this case, one simply measures the distance between the empirical cumulative distribution functions. This test rejects at high significance $(>99.99 \%)$ the null hypothesis that the distribution of $S_{3}$ and $S_{4}$ is Gaussian for the $R_{1}$ case. For $R_{2}$, the Gaussianity of $S_{4}$ is also rejected with similar confidence, but the null hypothesis is accepted $(P>35 \%)$ for the $S_{3}$ case.
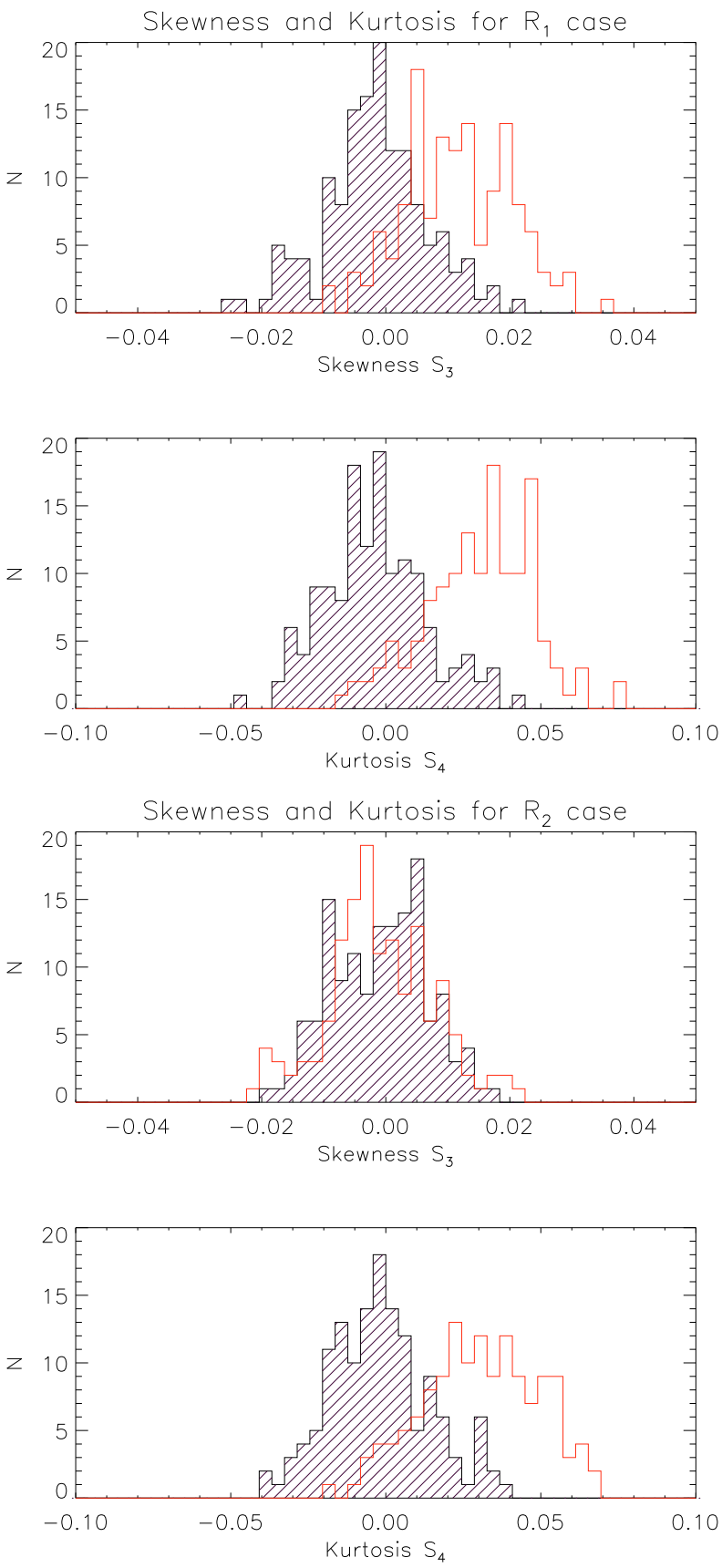

Fig. 8. Empirical observed probabilities for skewness $\left(S_{3}\right)$ and kurtosis $\left(S_{4}\right)$, sampled from 140 Monte Carlo noise maps (red). Shown are again the $R_{1}$ (top) and $R_{2}$ cases. The reference Gaussian sets are shown hatched. When comparing to the values reported in Table 2 it should be noted that the histograms here are computed over a considerably larger number of pixels.

In conclusion, the non-Gaussianity level induced by CR contamination in the large noise maps is weak and cannot be detected with high statistical significance even in the $R_{1}$ case, while the $R_{2}$ maps are, taken one at a time, indistinguishable from Gaussian maps, at least for the tests employed here. However, the situation changes when a set of maps is considered: our Monte Carlo analysis shows that, when a moderate number (140 in our case) are analyzed jointly, non-Gaussian signatures are detected even for $R_{2}$. As a consequence, an experiment that uses a similar detector but gathers significantly more data than considered here, may be prone to CR-induced non-Gaussianity, 
S. Masi et al.: Cosmic rays events in bolometric CMB observations

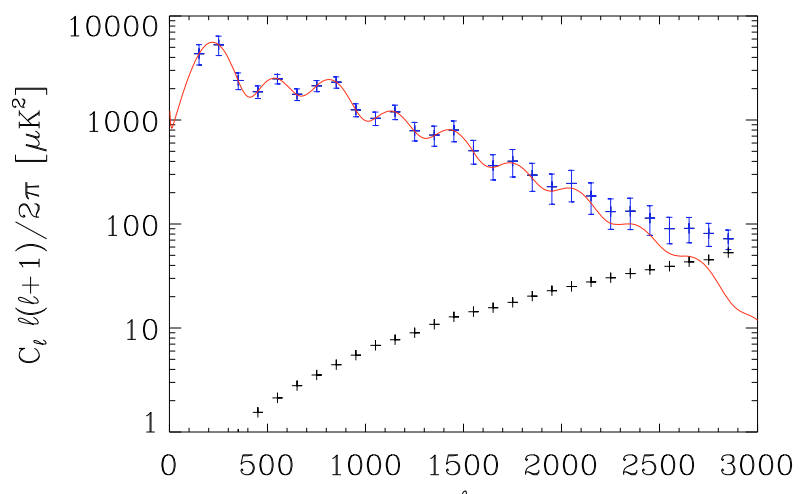

Fig. 9. Simulated measurement of the power spectrum of CMB anisotropy in a bolometric experiment affected by CR hits as in case $R_{1}$. The noise estimate neglects the presence of CR spikes. As a result, the spectrum is heavily biased (black crosses show this residual).

especially when high precision measurements are sought. This is especially critical for high sensitivity measurements of the $f_{\mathrm{NL}}$ parameter that quantifies the level of non-Gaussianity in the primordial perturbations (Bartolo et al. 2004). In particular, Planck (The Planck Collaboration 2006) is expected to tighten existing constraints on $f_{\mathrm{NL}}$ by roughly an order of magnitude. We leave a detailed study of the level of contamination by residual CR on $f_{\mathrm{NL}}$ estimates to a future paper.

\section{Biasing of power spectra}

The angular power spectrum of the CMB is a most valuable cosmological observable, which can be used to extract information about the underlying physical model. Thus, it is critical to assess the effect of contamination from residual, undetected spikes.

We thus estimate the angular power spectrum using cROMAster, a pseudo- $C_{\ell}$ estimator based on MASTER (Hivon et al. 2002), which was originally developed for (and applied to) BOOMERanG-B03 (Jones et al. 2006; Piacentini et al. 2006; Montroy et al. 2006; Masi et al. 2006) and later improved for PLANCK data analysis. cROMAster can estimate both auto- and cross-power spectra, where the former are more efficient but require noise removal and the latter are less efficient but are naturally unbiased (Polenta et al. 2005), therefore residual bias is not expected in the pure cross-spectrum case. We verified this assertion using simulations. The case of auto-spectra is more delicate since they require a companion Monte Carlo dataset to estimate and remove residual noise.

To verify the effect of the spikes, we firstly generated a Monte Carlo dataset that ignores the presence of residual spikes themselves, and only relies on the nominal detector noise level. When using this dataset as a noise estimate, the resulting power spectrum is heavily biased, as shown in Fig. 9.

Thus taking into account the effect of spikes in the Monte Carlo dataset is important. This can be done in two ways: the first and in principle more precise method is to process the Monte Carlo timelines by adding simulated spike signal with the same properties of the spikes seen in real data. When this is performed, a bias free auto-spectrum can be obtained as shown in Fig. 10.

However, this procedure relies on the knowledge of the underlying spike's rate and distribution. The spike characteristics and statistics can in principle be extrapolated from the data themselves, relying on the observed events. This procedure, however, significantly complicates the noise estimation and Monte Carlo

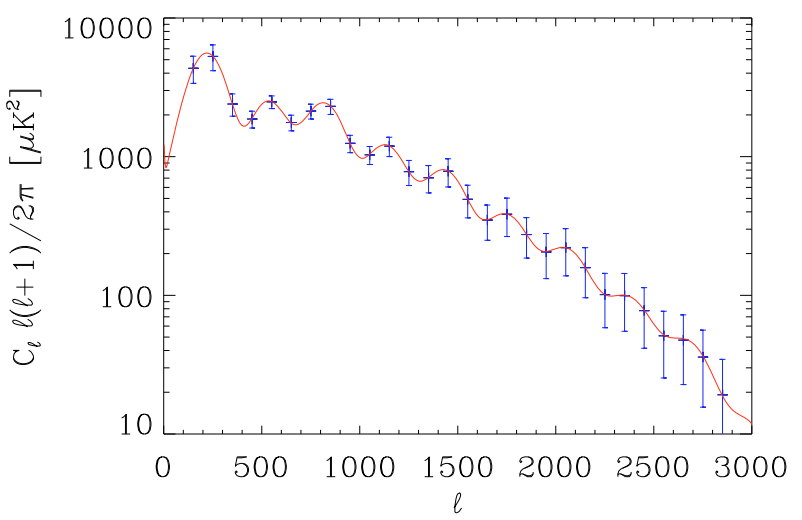

Fig. 10. Same as Fig. 9. Here the noise estimate accounts for CR spikes, assuming that their distribution is known a-priori. As a result, the spectrum is not biased.

generation pipeline of the experiment. Moreover, this approach is prone to biases, since the estimated statistics are derived from the most energetic CR events, which are detected above the noise, while the low energy ones, which contaminate the data, are not detected: their distribution can only be estimated by means of analytic extrapolation of the high energy distribution. The reliability is thus unknown. The variance produced by the extrapolated low-energy part of the distribution can be used to guess the importance of the problem. The only way to obtain robust estimates is to perform detailed and extensive Monte Carlo simulations (using packages such as GEANT-4, see Agostinelli et al. 2003) of the interaction of the full instrument with the cosmic environment. If the high-energy part of the simulated spectrum fits the data, the low energy part of the simulated spectrum can be used to estimate the noise bias from undetected spikes. This procedure is certainly complex and computationally very intensive.

We thus verified wether a simpler approach would produce sufficiently accurate results for power spectrum estimation. For this second scheme, we estimated the noise properties from the data that are contaminated by spikes but, in the timeline simulation, we avoided modeling the spike contribution and despiking procedure. In practice, we first measured the noise properties of the despiked timeline, after subtracting the signal contribution by means of standard iterative techniques (Ferreira \& Jaffe 2000), and used this dataset to estimate the noise power spectrum. Since a timeline contaminated by spikes exhibits some level of non-Gaussianity, the noise power spectrum does not encode all the information about our noise properties. However, we ignored this complication, and used the estimated spectrum information to generate stationary Gaussian realization of noise. Since the spike residual is probably non-Gaussian, this procedure is not strictly correct. However, as shown in Fig. 11, the residual bias we obtain is - visually - very small.

To quantify this statement, we performed the Hausman test (Polenta et al. 2005) on the band power we obtain from our simulations. The Hausman test is a powerful procedure to assess the significance of a residual noise bias in the spectrum. It uses both cross-spectra and auto-spectra estimates. We restrict ourselves to the $R_{1}$ and $R_{2}$ cases described above. As in Polenta et al. (2005), we define three test statistics to detect a bias in the noise estimation, $s_{1}=\sup _{r} B_{L}(r), s_{2}=\sup _{r}\left|B_{L}(r)\right|$, and $s_{3}=\int_{0}^{1} B_{L}^{2}(r) \mathrm{d} r$, where $B_{L}(r)$ is a random process defined as

$B_{L}(r)=\frac{1}{\sqrt{L}} \sum_{\ell=1}^{[L r]} H_{\ell}, r \in[0,1]$, 
A\&A 519, A24 (2010)

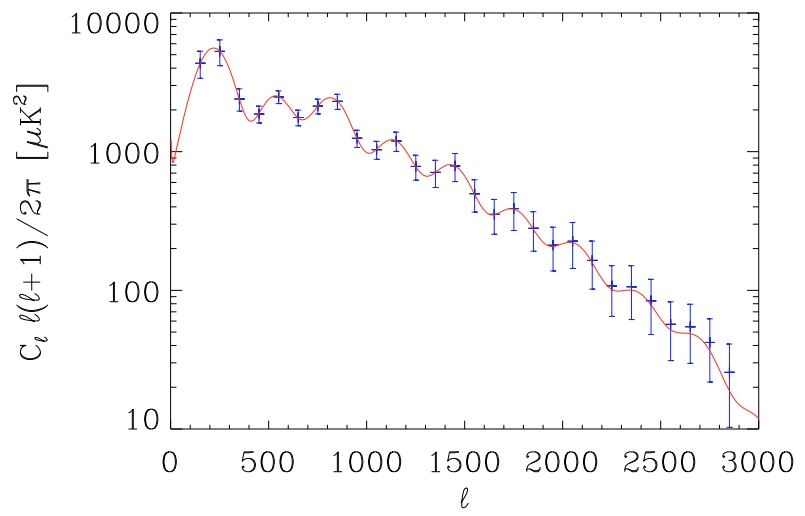

Fig. 11. Same as Fig. 9. Here the noise estimate has been performed from the dataset using a standard pipeline, i.e. subtracting the best fit signal contribution from the data timelines. The non-Gaussian nature of the residual spikes has been neglected. The residual bias in the power spectrum is small, and appears to be limited to the highest multipoles.

Table 3. Confidence level for bias detection with the Hausman test in cases $R_{1}$ (top three lines) and $R_{2}$ (bottom three lines), with a naively simulated noise estimation pipeline (see text).

\begin{tabular}{cccc}
\hline \hline & $68 \%$ & $95 \%$ & $99 \%$ \\
\hline$s_{1}$ & $100 \%$ & $96 \%$ & $87 \%$ \\
$s_{2}$ & $99 \%$ & $93 \%$ & $70 \%$ \\
$s_{3}$ & $96 \%$ & $69 \%$ & $39 \%$ \\
\hline$s_{1}$ & $59 \%$ & $19 \%$ & $10 \%$ \\
$s_{2}$ & $48 \%$ & $15 \%$ & $7 \%$ \\
$s_{3}$ & $38 \%$ & $8 \%$ & $1.4 \%$ \\
\hline
\end{tabular}

Notes. The parameters $s_{1}, s_{2}$, and $s_{3}$ represent three different bias estimators defined in Polenta et al. (2005) (see text).

where [.] denotes integer part, $L$ is the maximum multipole used in the harmonic analysis, and $H_{\ell}$ is the difference of the cross and auto (or in general unbiased versus biased) power spectrum estimators normalized by its variance. It can be shown that as $L \rightarrow \infty, B_{L}(r)$ converges to a Brownian motion process, whose properties are widely studied and well-known, and therefore can be used to test the null bias hypothesis. In this case, rather than relying on the asymptotical distribution, we used Monte Carlo simulations to draw the empirical distributions of the test statistics. Our results for the confidence of bias detection with $68 \%$, 95\%, and 99\% probability are shown in Table 3 .

We note that, despite being small, the bias can always be detected at a high statistical significance. This is a strong indication that the noise estimation pipeline for an high precision experiment must account for the presence of spikes in a more accurate way than the simple procedure set forth above, at least if auto spectra are desired.

While we do not explicitly simulate an experiment capable of measuring polarization spectra, it is clear that the same conclusions hold, a fortiori, when linear polarization can be measured. On the other hand, a spectral pipeline that relies only on cross-spectra is very robust to the effect of spikes.

Hence, cross-spectra, while being less efficient than autospectra, are certainly more adequate for an experiment contaminated by cosmic rays, at least as long as correlated events between different detectors are excluded.

\section{Conclusions}

Bolometric observations carried out from space are affected by cosmic rays. The data must be despiked to be used efficiently and avoid serious biases in the results. Pixel-based outliers removal works well: the power spectra of the despiked timelines closely resemble the original Gaussian noise used in the simulation. However, low-level events remain hidden in the noise, resulting in a positive shift of the average signal measured in each pixel, and increasing its variance.

The maps obtained from despiked data are non-Gaussian. The level of non-Gaussianity depends on the rate of the spikes, on the deposited energy and on the time constant and noise of the detectors. Using the skewness and the kurtosis of the pixel temperatures as simple non-Gaussianity indicators, we have found that, in the case of the 100 square degree survey, the CR-induced non-Gaussianity is unlikely to be detected if spider-web bolometers are used, but would be marginally detectable for slow membrane detectors. For experiments with longer integration times and/or lower noise detectors, the CR-induced non-Gaussianity will be significant. This will probably affect the constraints on the primordial non-Gaussianity expected by present space-borne missions. Detailed studies need to be performed in these cases.

Using the standard analysis pipeline on the map produced by the despiked timeline, we have found that the residual hidden events produce a positive bias in the angular power spectrum of the map at high multipoles. In the specific example of the 100 square degree survey, the expected bias is negligible for spider-web bolometers, but can be important for slow membrane detectors. However, since all modern experiments use bolometer arrays, cross-spectra can be computed in place of auto-spectra. These spectra are virtually unaffected by the CR-induced bias problem. The only unavoidable problem however is a significant decrease in the effective integration time and redundancy of the maps, in the case of slow membrane detectors.

Acknowledgements. This work has been supported by Italian Space Agency contracts "COFIS", "Planck-HFI" and "OLIMPO" and by PRIN 2006 "Cosmologia Millimetrica con Grandi Mosaici di Rivelatori" of the Ministero dell'Istruzione, dell'Università e della Ricerca.

\section{References}

Agostinelli, S., Allison, J., Amako, K., et al. 2003, Nucl. Inst. and Methods A, 506,250

Bartolo, N., Komatsu, E., Matarrese, S., \& Riotto, A. 2004, Phys. Rep., 402, 103

Billot, N., Rodriguez, L., Okumura, K., Sauvage, M., \& Agnèse, P. 2009, Astrophysics Detector Workshop 2008, ed. P. Kern, EAS Publ. Ser., 37, 119

Carlstrom, J. E., Ade, P. A. R., Aird, K. A., et al. 2009, PASP, submitted [arXiv: 0907.4445]

Caserta, A., de Bernardis, P., Masi, S., \& Mattioli, M. 1990, Nuclear Instrumentation and Methods in Physics Research A294, 328

Crill, B. P., Ade, P. A. R., Battistelli, E. S., et al. 2008, Space Telescopes and Instrumentation 2008: Optical, Infrared, and Millimeter, ed. J. M. Oschmann, Jr., M. W. M. de Graauw, \& H. A. MacEwen, Proc. SPIE, 7010, [arXiv: 0807.1548]

De Troia, G., Ade, P. A. R., Bock, J. J., et al. 2003, MNRAS, 343, 284

Ferreira, P. G., \& Jaffe, A. H. 2000, MNRAS, 312, 89

Hinderks, J. R., Ade, P., Bock, J., et al. 2009, ApJ, 692, 1221

Hivon, E., Górski, K. M., Netterfield, C. B., et al. 2002, ApJ, 567, 2

Holmes, W. A., Bock, J. J., Crill, B. P., et al. 2008, Appl. Opt., 47, 5996

Jones, W. C., Bhatia, R. S., Bock, J. J., \& Lange, A. E. 2003, Proc. SPIE, 4855, 227

Jones, W. C., Ade, P. A. R., Bock, J. J., et al. 2006, ApJ, 647, 823

Kaiser, N., \& Stebbins, A. 1984, Nature, 310, 391

Komatsu, E., Smith, K. M., Dunkley, J., et al. 2010, ApJS, submitted [arXiv: 1001.4538] 
Kuo, C. L. 2006, Nuclear Instruments and Methods in Physics Research A, 559 , 608

Komatsu, E., Kogut, A., Nolta, M. R., et al. 2003, ApJS, 148, 119

Macias-Perez, J. F., Lagache, G., Maffei, B., et al. 2007, A\&A, 467, 1313

Marsden, G., Ade, P. A. R., Benton, S., et al. 2008, SPIE Conf. Proc. [arXiv:0805.4420]

Masi, S., Ade, P. A. R., Bock, J. J., et al. 2006, A\&A, 458, 687

Mauskopf, P. D., Bock, J. J., Del Castillo, H., Holzapfel, W. L., \& Lange, A. E. 1997, Appl. Opt., 36, 4

Montroy, T. E., Ade, P. A. R., Bock, J. J., et al. 2006, ApJ, 647, 813

Nati, F., Ade, P., Boscaleri, A., et al. 2007, New Astron. Rev., 51, 385

Natoli, P., De Troia, G., Hikage, C., et al. 2010, MNRAS, in press [arXiv:0905.4301]

Nolta, M. R., Dunkley, J., Hill, R. S., et al. 2009, ApJS, 180, 296

Oxley, P., Ade, P. A., Baccigalupi, C., et al. 2004, Proc. SPIE Int. Soc. Opt. Eng., 5543, 320

Pascale, E., Ade, P. A. R., Bock, J. J., et al. 2008, ApJ, 681, 400

Piacentini, F., Ade, P. A. R., Bock, J. J., et al. 2006, ApJ, 647, 833
Polenta, G., Marinucci, D., Balbi, A., et al. 2005, JCAP, 0511, 001

Press, W. H., Flannery, B. P., Teukolsky, S. A., \& Vetterling, W. T. 1992, Numerical Recipes in FORTRAN, The Art of Scientific Computing, 2nd edn (Cambridge: Cambridge University Press)

Samtleben, D. 2007, Nuovo Cimento, 122B, 1353

Sayers, J., Golwala, S. R., Rossinot, P., et al. 2009, ApJ, 690, 1597

Schultz, B., Bock, J. J., Lu, N., et al. 2008, in Millimeter and Submillimeter Detectors and Instrumentation for Astronomy IV, ed. W. D. Duncan, W. S. Holland, S. Withington, \& J. Zmuidzinas, Proc. SPIE, 7020, 52

Siringo, G., Kreysa, E., Kovács, A., et al. 2009, A\&A, 497, 945

Swetz, D. S., Ade, P. A. R., Allen, C., et al. 2008, Proc. SPIE, 7020, 6

The PLANCK Collaboration 2006, unpublished, [arXiv: astro-ph/0604069]

Verde, L., Wang, L., Heavens, A. F., \& Kamionkowski, M. 2000, MNRAS, 313, 141

Wilson, G. W., et al. 2008, MNRAS, 385, 2225

Yoon, K. W., Ade, P. A. R., Barkats, D., et al. 2006, in Millimeter and Submillimeter Detectors and Instrumentation for Astronomy III, Proc. SPIE, 6275, [arXiv: astro-ph/0606278] 